Article

\title{
Green Tea Polyphenol (-)-Epigallocatechin Gallate (EGCG) Attenuates Neuroinflammation in Palmitic Acid-Stimulated BV-2 Microglia and High-Fat Diet-Induced Obese Mice
}

\author{
Limin Mao, Danielle Hochstetter, Liyun Yao, Yueling Zhao, Jihong Zhou, Yuefei Wang * and \\ Ping $X u^{*}$ \\ Tea Research Institute, Zhejiang University, Hangzhou 310058, China; maolm@zjtea.com (L.M.); \\ 21816158@zju.edu.cn (D.H.); 21716065@zju.edu.cn (L.Y.); 11616050@zju.edu.cn (Y.Z.); \\ zhoujihong@zju.edu.cn (J.Z.) \\ * Correspondence: zdcy@zju.edu.cn (Y.W.); zdxp@zju.edu.cn (P.X.); Tel.: +86-0571-8898-2263 (Y.W.); \\ +86-0571-8898-2217 (P.X.)
}

Received: 3 September 2019; Accepted: 11 October 2019; Published: 13 October 2019

\begin{abstract}
Obesity is closely associated with neuroinflammation in the hypothalamus, which is characterized by over-activated microglia and excessive production of pro-inflammatory cytokines. The present study was aimed at elucidating the effects of (-)-epigallocatechin gallate (EGCG) on palmitic acid-stimulated BV-2 microglia and high-fat-diet-induced obese mice. The results indicated the suppressive effect of EGCG on lipid accumulation, pro-inflammatory cytokines (TNF- $\alpha$, IL-6, and IL-1 $\beta$ ) release, and microglial activation in both cellular and high-fat-diet rodent models. These results were associated with lower phosphorylated levels of the janus kinase 2/signal transducers and activators of transcription 3 (JAK2/STAT3) signaling pathway. In conclusion, EGCG can attenuate high-fat-induced hypothalamic inflammation via inhibiting the JAK2/STAT3 signaling pathways in microglia.
\end{abstract}

Keywords: tea polyphenols; saturated fatty acids; obesity; hypothalamus; inflammation

\section{Introduction}

The prevalence of obesity is dramatically increasing worldwide in recent decades [1]. The consumption of high-fat diet (HFD) is a causative factor for being overweight or obese [2]. HFD is rich in saturated fatty acids (SFA), such as palmitic acid (PA), stearic acid, and lauric acid. An excess amount of SFA in the diet is a key pathogenic link between HFD-induced obesity and concomitant chronic low-grade systemic inflammation [3].

The hypothalamus section of the central nervous system (CNS) regulates lipid homeostasis and energy metabolism [4-6]. Several hypothalamic nuclei, namely the arcuate nucleus (ARC) and the paraventricular nucleus (PVN), are involved in maintaining energy balance [7]. Microglia are the major macrophages in the hypothalamus, which can initiate rapid morphological and functional transformations in response to excessive dietary SFA, triggering the abundant release of inflammatory cytokines, followed by a series of inflammatory reactions [8]. Lately, anti-inflammatory therapies based on the microglia activation process have shown high prospects for the treatment of obesity and metabolic syndromes [6].

Green tea, a widely consumed beverage, contains many bioactive constituents, among which (-)-epigallocatechin gallate (EGCG) is one of the most potent compounds with anti-obesity effects $[9,10]$. Many studies have reported that EGCG increases energy expenditure, alleviates adipose insulin 
resistance, induces adipocyte apoptosis, and inhibits preadipocyte differentiation [11]. Nevertheless, a detailed central modulation mechanism of EGCG on HFD-induced neuroinflammation has not yet been elucidated.

In the present study, the influence of EGCG was evaluated on SFA-mediated lipid accumulation and microglia-mediated hypothalamic inflammation in PA-stimulated BV-2 cells and HFD-induced obese mice. Our results show that EGCG attenuates HFD-induced neuroinflammation via suppressing the JAK2/STAT3 signaling pathway. This outcome would provid insight into the neurological mechanism of the anti-obesity activity of EGCG.

\section{Results}

\subsection{EGCG Attenuates Lipid Accumulation and Inflammatory Responses in PA-Stimulated BV-2 Cells}

PA induces lipid accumulation and pro-inflammatory cytokine expression in microglia [12]. We first investigated whether EGCG could reduce lipid accumulation in PA-stimulated BV-2 cells with oil red O staining. The tested concentrations of PA and EGCG in all the experiments were subjected to the MTT (3-(4,5)-dimethylthiahiazo (-z-y1)-3,5-di- phenytetrazoliumromide) assay, and EGCG at concentrations ranging from 5 to $20 \mu \mathrm{M}$ did not affect the cell viability (data not shown). According to Figure 1a, oil red O staining revealed that the accumulation of lipid droplets in BV-2 cells was increased substantially after PA treatment, which is clearly improved in groups with EGCG pretreatment. In addition, ELISA showed lower levels of TNF- $\alpha$, IL- 6 , and IL- $1 \beta$ in the EGCG pretreated groups than the PA group, and with the increase of EGCG concentration, the activity increased (Figure 1b). These data suggest that EGCG suppressed the PA-induced excessive lipid accumulation and inflammatory response in microglia in a dose-dependent manner. In addition, the concentration gradient assay suggested that all three doses of EGCG could attenuate the PA-induced excessive lipid accumulation and inflammatory responses, while the highest dose produced the most effective inhibition.
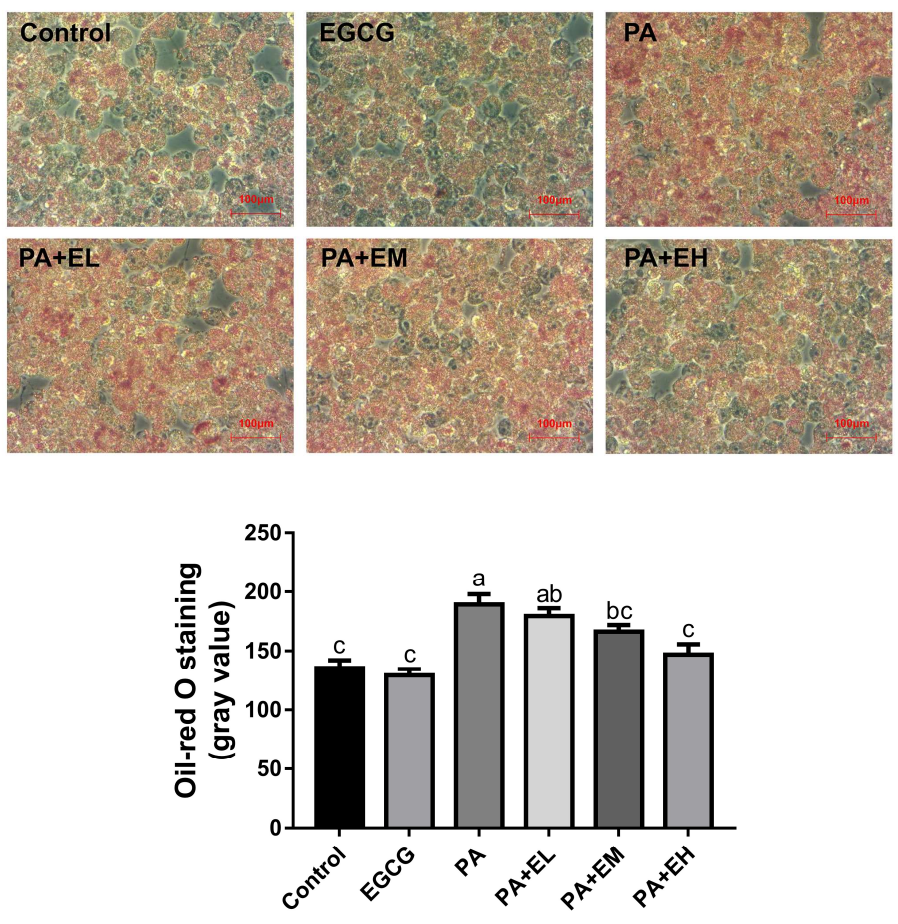

(a)

Figure 1. Cont. 

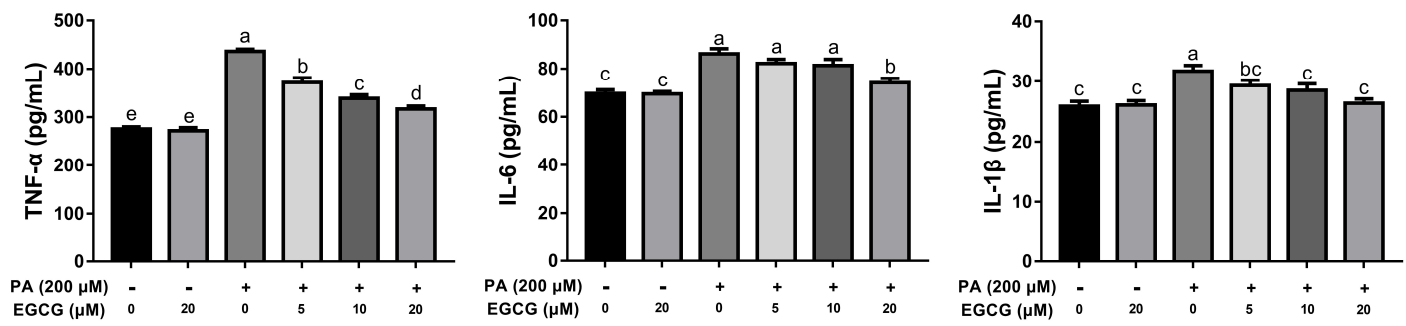

(b)

Figure 1. Effects of (-)-epigallocatechin gallate (EGCG) on lipid accumulation and inflammatory responses of PA-stimulated BV-2 cells. BV2 cells were pretreated with different dosages of EGCG (EL: low concentration of EGCG $(5 \mu \mathrm{M})$, EM: medium concentration of EGCG $(10 \mu \mathrm{M})$, EH: high concentration of EGCG $(20 \mu \mathrm{M}))$ for $2 \mathrm{~h}$ and then stimulated with $200 \mu \mathrm{M}$ palmitic acid (PA) for $24 \mathrm{~h}$. (a) Lipid distribution in BV-2 cells was observed by oil red O staining and analyzed with Imagej; lipids were stained red; (b) Levels of TNF- $\alpha$, IL-6, and IL-1 $\beta$ in the conditioned medium were measured by ELISA. Data are means \pm SEM of three independent experiments performed in triplicate. Different superscript letters indicate significantly different means at $p<0.05(\mathrm{a}>\mathrm{b}>\mathrm{c}>\mathrm{d})$.

\subsection{EGCG Suppresses Phosphorylation of JAK2 and STAT3 in PA-Stimulated BV-2 Cells}

Phosphorylation of STAT3 can be induced by JAK2, and inactivation of STAT3 triggers an inflammatory response [13,14]. The levels of related proteins were evaluated for investigating whether EGCG inhibited the neuroinflammation via the JAK2/STAT3 pathway. The western blot analysis (Figure 2) demonstrated that the phosphorylation of JAK2 and STAT3 were significantly upregulated by PA compared to the control group, whereas EGCG prevented the phosphorylation of JAK2 and STAT3 in PA-stimulated BV-2 cells. These data establish the JAK2/STAT3 pathway as an effective target for EGCG in PA-stimulated BV-2 cells.
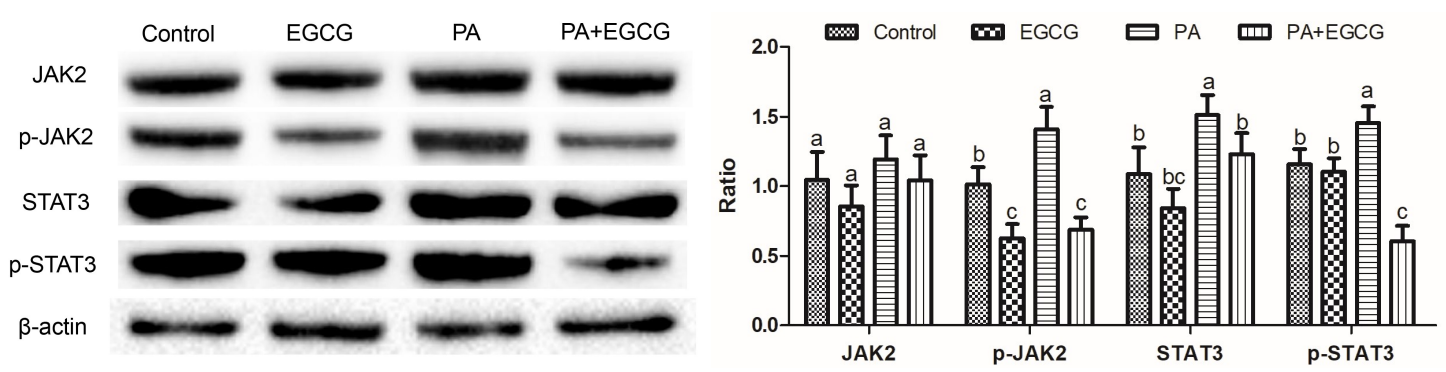

Figure 2. Inhibition effect of (-)-epigallocatechin gallate (EGCG) on JAK2/STAT3 signaling activation in PA-stimulated BV-2 cells. BV-2 cells were pretreated with $20 \mu \mathrm{M}$ EGCG for $2 \mathrm{~h}$ and then stimulated with $200 \mu \mathrm{M}$ palmitic acid for $24 \mathrm{~h}$. Representative Western blots for JAK2 and STAT3 phosphorylation in BV-2 cells were shown. Data are means \pm SEM of three independent experiments performed in triplicate. Different superscript letters indicate significantly different means at $p<0.05(\mathrm{a}>\mathrm{b}>\mathrm{c})$.

\subsection{EGCG Ameliorates HFD Induced Obesity}

In the present study, obesity in male C57BL/6J mice was induced by HFD during an 8-week period. EGCG-treated (fed with a $60 \mathrm{kcal} \%$ high-fat diet supplemented with 1\% EGCG) mice showed an obvious reduction in body weight, lipid deposition, and epididymal adipocytes size (Figure 3a-c). 


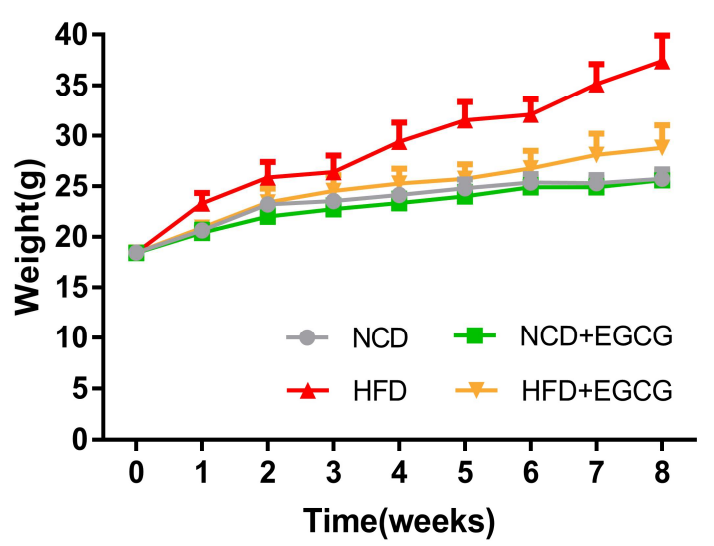

(a)

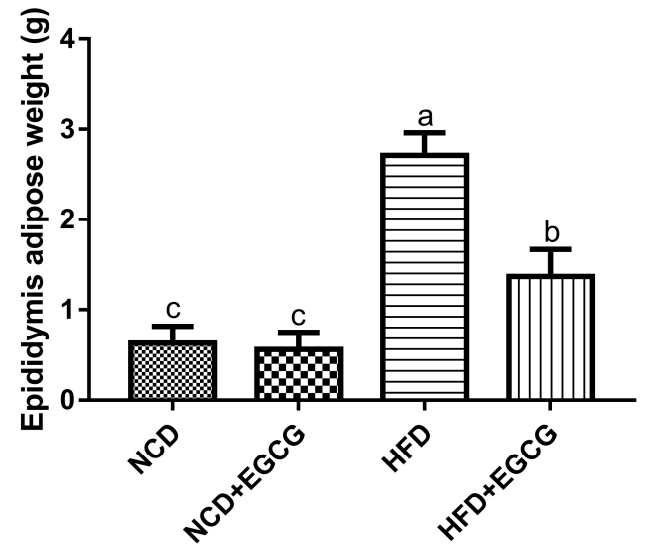

(b)
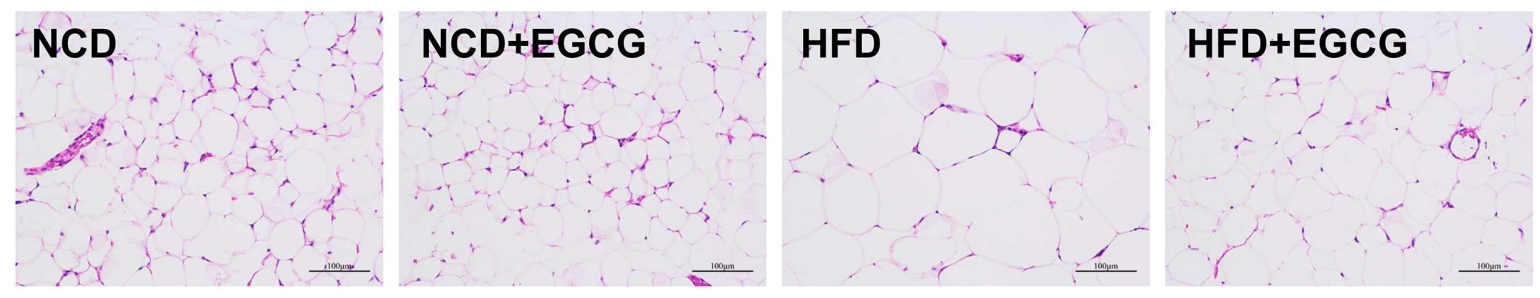

(c)

Figure 3. Effect of (-)-epigallocatechin gallate (EGCG) on HFD-induced obesity. (a) Weight variation tendency of different groups; (b) Weight of epididymis adipose; (c) Representative hematoxylin and eosin (H\&E) staining of epididymis adipose sections. Data are means \pm SEM $(n=6)$. Different superscript letters indicate significantly different means at $p<0.05(\mathrm{a}>\mathrm{b}>\mathrm{c})$.

Serum glucose and lipid levels were also measured. Compared to HFD mice, EGCG treated group significantly restored the blood glucose, total cholesterol (TC), and triglyceride (TG) levels under fasting conditions (Table 1). These results illustrate the anti-obesity potential of EGCG.

Table 1. Effects of (-)-epigallocatechin gallate (EGCG) on serum biochemical parameters.

\begin{tabular}{ccccc}
\hline Serum Biochemical Indices & NCD & NCD + EGCG & HFD & HFD + EGCG \\
\hline Glucose (mmol/L) & $5.93 \pm 0.57^{\mathrm{c}}$ & $6.02 \pm 0.48^{\mathrm{c}}$ & $8.82 \pm 0.66^{\mathrm{a}}$ & $6.78 \pm 0.34^{\mathrm{b}}$ \\
TG (mmol/L) & $1.16 \pm 0.15^{\mathrm{b}}$ & $1.24 \pm 0.11^{\mathrm{b}}$ & $1.38 \pm 0.14^{\mathrm{a}}$ & $1.15 \pm 0.19^{\mathrm{b}}$ \\
TC (mmol/L) & $3.10 \pm 0.25^{\mathrm{c}}$ & $2.91 \pm 0.15^{\mathrm{c}}$ & $4.36 \pm 0.43^{\mathrm{a}}$ & $3.44 \pm 0.16^{\mathrm{b}}$ \\
HDL (mmol/L) & $2.96 \pm 0.34^{\mathrm{a}}$ & $2.75 \pm 0.16^{\mathrm{a}}$ & $2.89 \pm 0.29^{\mathrm{a}}$ & $3.14 \pm 0.30^{\mathrm{a}}$ \\
LDL (mmol/L) & $0.31 \pm 0.09^{\mathrm{a}}$ & $0.29 \pm 0.03^{\mathrm{a}}$ & $0.39 \pm 0.11^{\mathrm{a}}$ & $0.24 \pm 0.08^{\mathrm{a}}$ \\
\hline
\end{tabular}

NCD, a normal chow diet; NCD + EGCG, a normal chow diet supplemented with 1\% EGCG; HFD, a $60 \mathrm{kcal} \%$ high-fat diet; HFD + EGCG, a $60 \mathrm{kcal} \%$ high-fat diet supplemented with $1 \%$ EGCG. Data are presented as mean \pm $\operatorname{SEM}(n=6)$. Superscript letters $\mathrm{a}, \mathrm{b}$ and $\mathrm{c}$ indicate significantly different means at $p<0.05(\mathrm{a}>\mathrm{b}>\mathrm{c})$.

\subsection{EGCG Alleviates the Obesity-Associated Neuroinflammation of the Hypothalamus}

The hypothalamus centrally controls the energy homeostasis. Recent studies have revealed the involvement of chronic low-grade hypothalamic inflammation in the modulation of DIO (diet-induced obesity) $[15,16]$. In our study, the EGCG-treated mice presented a marked decline of the inflammatory cytokine levels (TNF- $\alpha$, IL-6, and IL-1 $\beta$ ) in the hypothalamus than the HFD group (Figure 4a,b). The molecular underlying mechanisms related to these changes were elucidated through western blot analysis, which measured the expression of the key factors in the JAK2/STAT3 pathway. Figure $4 \mathrm{~b}$ shows that the phosphorylation of JAK2 and STAT3 was noticeably upregulated by HFD, which could 
be suppressed by EGCG. Thus, EGCG can alleviate the obesity-associated neuroinflammation of the hypothalamus via regulating JAK2/STAT3 signaling pathway.

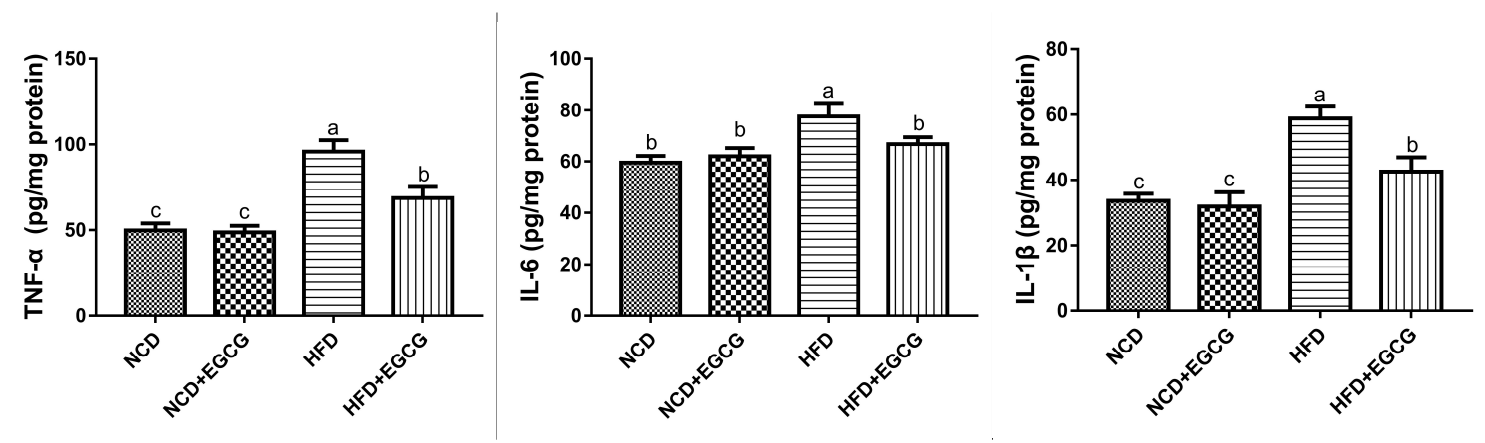

(a)

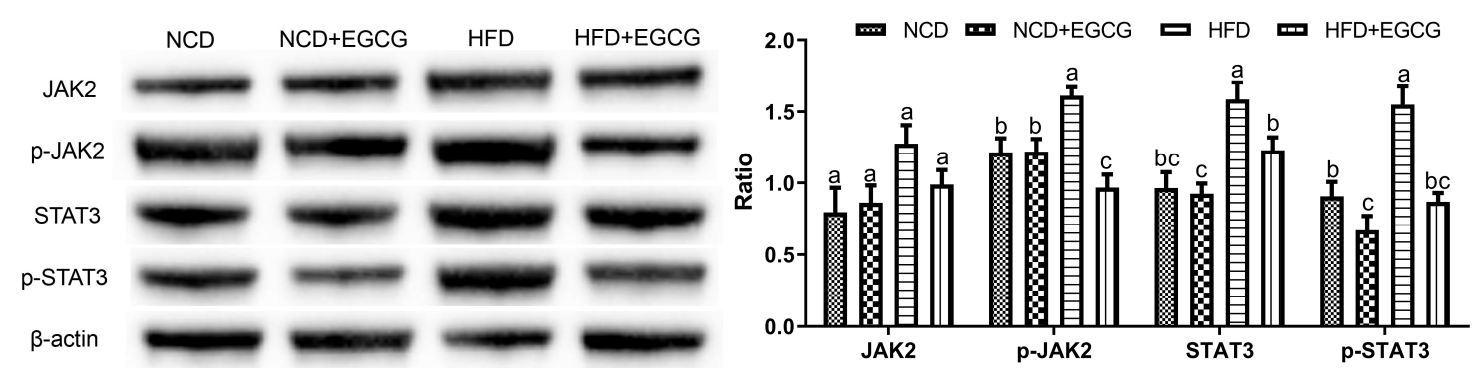

(b)

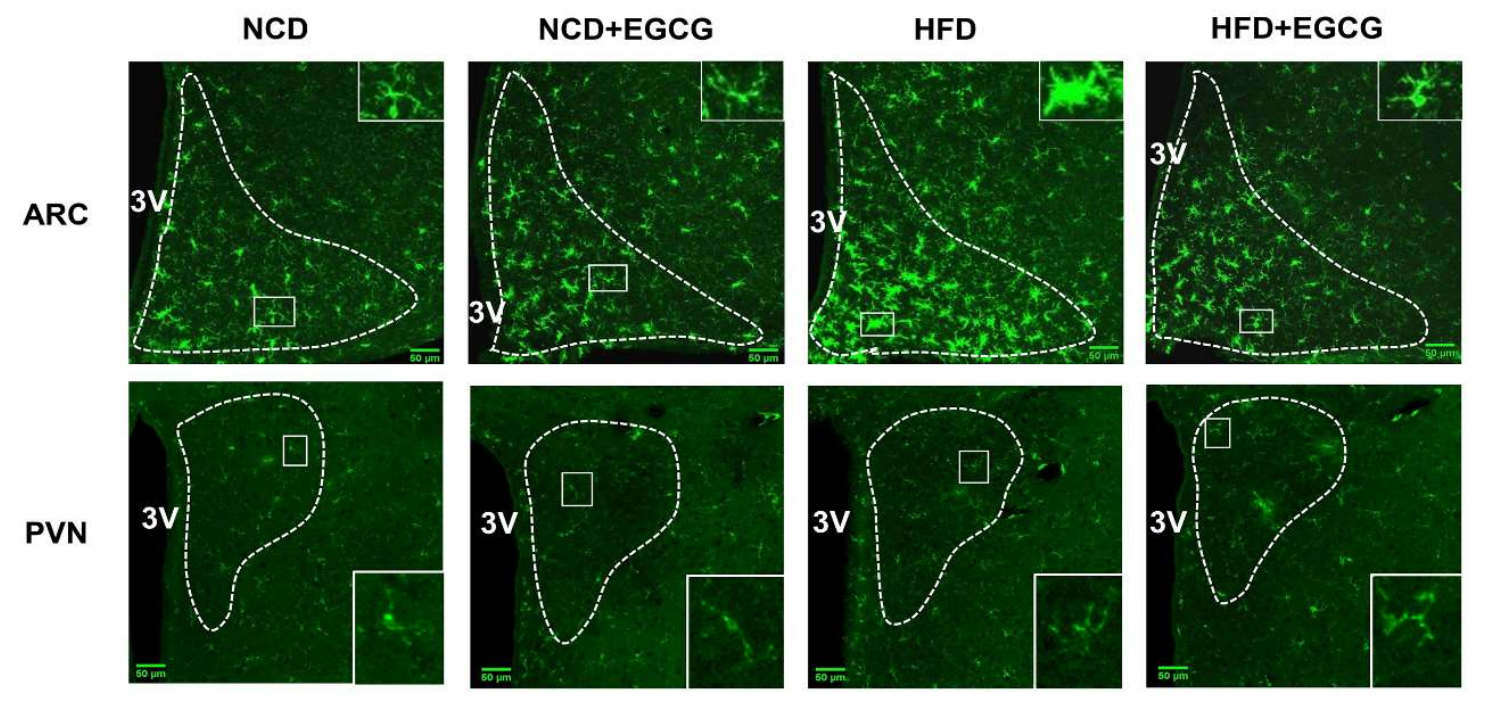

(c)

Figure 4. Inhibition effect of (-)-epigallocatechin gallate (EGCG) on obesity-associated neuroinflammation of hypothalamus. (a) Concentrations of TNF- $\alpha$, IL-6, IL- $1 \beta$ in the hypothalamus; (b) Representative Western blots for JAK2 and STAT3 phosphorylation levels in the hypothalamus; (c) Representative micrographs of immunofluorescence labeling for Iba1 in the hypothalamic arcuate nucleus (ARC) and paraventricular nucleus (PVN) (outlined by white dashed lines) and higher magnification insets (outlined by white solid lines). Data are means \pm SEM of three independent experiments performed in triplicate $(n=3)$. Different superscript letters indicate significantly different means at $p<0.05(\mathrm{a}>\mathrm{b}>\mathrm{c})$. 
Microglia activation is associated with the development of obesity-induced hypothalamic inflammation [17,18]. EGCG induced inhibition of microglial activation in hypothalamic ARC and PVN was identified by evaluating Iba-1 (a microglial marker) immunofluorescence labeling. Previous studies have identified two major morphological types of microglia, namely "ramified resting" and "activated amoeboid", which are associated with the proinflammatory, cytotoxic, and immunoregulatory functions [19]. Ramified microglia with numerous thin, long processes are known to transit into rounded soma with little or no branching when stimulated under pathological conditions [20]. Figure 4c shows a significant increase in Iba-1 labeling with amoeboid morphological transitions in the hypothalamic ARC of HFD mice. Conversely, many quiescent microglial cells with small cell bodies and ramified processes were observed in the hypothalamic ARC of control and EGCG-treated mice (Figure 4c). In addition, no significant difference in microglial activation was observed in PVN (Figure 4c).

To sum up, dietary supplementation with EGCG effectively alleviated obesity-associated hypothalamic inflammation mediated via down-regulation of the JAK2/STAT3 signaling pathway, which is most likely related to the microglial inflammatory signaling in hypothalamic ARC instead of PVN.

\section{Discussion}

Many studies support that tea polyphenols play as an effective role in lowering the risk of obesity, diabetes, and metabolic syndrome. Several mechanisms have been proposed, such as reducing adipocyte proliferation, impeding the absorption of fat, and promoting the oxidation of fatty acids [21-24]. Our previous studies have shown that EGCG ameliorated HFD-induced obesity without affecting the food and energy intake, suggesting that EGCG might play an important role on promoting energy expenditure [25]. The central nervous system, particularly the hypothalamus, is the major regulator of energy metabolism. However, the role of EGCG in regulating the central nervous system in HFD-induced obesity with a detailed underlying molecular mechanism remains unclear.

Inflammatory responses and dysfunction in the hypothalamus are the common features of HFD-induced obesity models [26-28]. Microglia participates in a cell-mediated hypothalamic immune response to dietary excess; though, the mechanisms by which microglia inflicts neuronal injury and functional disorder should be thoroughly studied $[18,29,30]$. Palmitic acid is a common SFA in human diets, which is accumulated in the hypothalamus after HFD consumption [12,31]. Based on the previous method [32], PA-stimulated BV-2 cells were utilized for investigating the effect of EGCG on HFD-induced microglial inflammation. EGCG diminished the excessive lipid accumulation and the production of proinflammatory cytokines in this model. These results were also confirmed in the subsequent HFD-induced obese mice model. Also, EGCG inhibited the activation of microglia in the hypothalamic ARC. Consistent with our previous study [25], these data suggest that EGCG ameliorated metabolic disorders via reducing hypothalamic inflammatory responses, and microglial-induced central sensitization in the hypothalamic ARC. Hypothalamic ARC has been regarded as one of the core control centers of metabolism, in which proopiomelanocortin (POMC) and agouti-related neuropeptide (AgRP) neurons respond to signaling cues from the periphery, such as leptin, insulin, or ghrelin [33]. In previous studies, chronic low-grade hypothalamic inflammation was proved to disrupt peripheral insulin and leptin signaling and glucose homeostasis [34,35]. The neurological mechanism of EGCG in controlling obesity may be related to the regulation of these neuropeptides and related hormones.

STAT3 is a representative of signal transducer and activator of transcription (STAT) family, which can be phosphorylated by Janus kinase (JAK) in response to ligand (LPS, PA, etc.) stimulation [36]. Phosphorylation of STAT3 is closely related with inflammation, oxidative stress, and apoptosis [37]. Recent studies have proposed that proinflammatory responses in microglia are controlled by the upstream JAK2/STAT3 pathway, which is involved in the central regulation of hypothalamic inflammation in high-fat-diet-induced obese rodents [14,36,38-41]. Several studies have reported that plant polyphenols, such as Ougan polymethoxyflavones [40], proanthocyanidins [42], and green 
tea polyphenol [25], could reduce neuroinflammation through the JAK2/STAT3 pathway. Consistent with previous studies, our results demonstrate that EGCG decreases the abnormally elevated protein expression of p-JAK2 and its downstream factor p-STAT3 induced by the HFD, and then time activated STAT3 translocates into the nucleus for regulating the expression of inflammatory-related target genes [43]. These results indicate that EGCG was involved in the regulation of the JAK2/STAT3 signaling pathway negative feedback, or the activation of negative regulators of the JAK2/STAT3 signaling pathway.

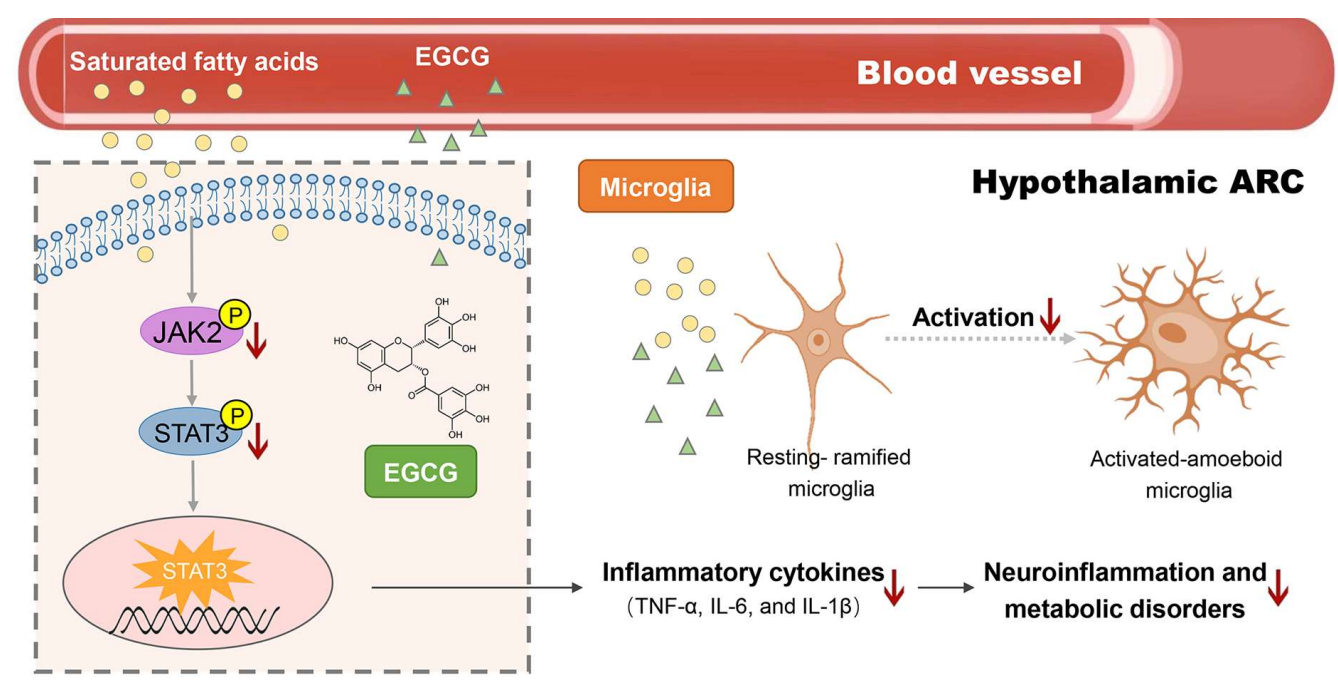

Figure 5. Possible neurological mechanisms of (-)-epigallocatechin gallate (EGCG) on HFD-induce obesity.

In summary, the present study demonstrated that EGCG decreased the HFD-induced neuroinflammation and microglial activation in the hypothalamic ARC region, which may be actualized via altered JAK2/STAT3 signaling in the hypothalamus (Figure 5). These findings elucidate the neurological mechanism of the anti-obesity effect of EGCG, suggesting that EGCG might be a potential therapeutic agent for the treatment of obesity-mediated neuroinflammation. Specific chemical inhibitors for the JAK2/STAT3 pathway could be used in cell models and mice models to confirm further if such changes in cytokine levels and microglial activation levels are dependent on JAK2/STAT3 signaling upon EGCG treatment. Meanwhile, more research is required for elucidating the effects of EGCG on the interaction of microglia, astrocytes, related neuropeptide, and peripheral hormone signaling in hypothalamic inflammation for further clarification of the precise neuronal networks involved in metabolic regulation.

\section{Materials and Methods}

\subsection{Materials}

EGCG (purity $>95 \%$ ) was obtained from Huzhou Rongkai Foliage Extract Co., Ltd. (Huzhou, China). A fatty acid-albumin complex solution containing palmitic acid (PA, Sigma-Aldrich, St. Louis, MO, USA) and fatty acid-free bovine serum albumin (BSA, Sigma, St Louis, MO, USA) was prepared as described previously [44]. All cell culture reagents were purchased from Gibco (Grand Island, NY, USA).

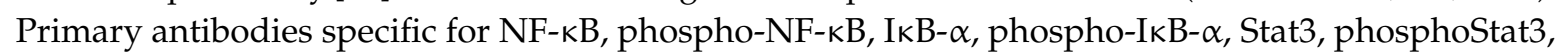
Jak2, and phospho-Jak2 were purchased from Cell Signaling Technology (Beverly, MA, USA), and Iba-1 was obtained from Wako Pure Chemical Industries, Ltd. (Osaka, Japan). D12450J normal chow diet and D12492 high-fat diet were purchased from Research Diets, Inc. Co., Ltd. (New Brunswick, NJ, USA). 


\subsection{BV-2 Cell Culture and Treatment}

The mouse microglial cell line BV-2 (ATCC, Rockville, MD, USA) was routinely cultured in Roswell Park Memorial Institute (RPMI) 1640 medium, supplemented with 10\% fetal bovine serum (FBS), $100 \mathrm{U} / \mathrm{mL}$ penicillin, $100 \mathrm{mg} / \mathrm{mL}$ streptomycin and $2 \mathrm{mM} \mathrm{L-glutamine} \mathrm{at} 37^{\circ} \mathrm{C}$ in a humidified $5 \%-\mathrm{CO}_{2}$ incubator. In all experiments, the cells were seeded at a density of $1 \times 10^{5} \mathrm{cells} / \mathrm{mL}$. After seeding for $24 \mathrm{~h}$, the culture medium was replaced with serum-free RPMI (Roswell Park Memorial Institute) 1640 medium, pretreated with different doses of EGCG $(5,10,20 \mu \mathrm{M})$ for $2 \mathrm{~h}$, and finally incubated with $200 \mu \mathrm{M}$ PA. After $24 \mathrm{~h}$ of PA treatment, the growth medium was collected for detecting the inflammatory cytokines. Cellular proteins were extracted for further western blot analysis.

\subsection{Oil Red O Staining}

BV-2 cells were fixed with $10 \%$ formaldehyde in PBS for $10 \mathrm{~min}$ and then stained with fresh oil red O solution (Solarbio, Beijing, China) for $10 \mathrm{~min}$ at room temperature. After washing with distilled water, the stained cells were inspected under a microscope (Zeiss, Oberkochen, Germany). The droplets were subsequently dissolved in isopropanol, and the lipid content was quantified by measuring the absorbance at $490 \mathrm{~nm}$. ImageJ software (NIH, Bethesda, MD, USA) was applied for analyzing the gray value.

\subsection{Animals}

All animals were handled following the guidelines of the National Institutes of Health (NIH) for animal care and the use of laboratory animals. Also, the animal experiments were approved by the Animal Care and Use Committee at Zhejiang University (ethic approval code: ZJU20190071). Twenty four-week-old male C57BL/6J mice were purchased from Shanghai SLAC Laboratory Animal Co., Ltd. (Shanghai, China). The mice were randomly assigned to four groups $(n=6 /$ group) with free access to food and water in standardized conditions (12:12 h of the light-dark cycle, the temperature of $22 \pm 2{ }^{\circ} \mathrm{C}$ with a relative humidity of $55 \pm 5 \%$ ). After a one-week-adaptation, four groups of mice were fed on the following diets for eight weeks: a normal chow diet (NCD, with $10 \%$ of energy from fat), a normal chow diet blending with 1\% EGCG (NCD + EGCG, with 10\% of energy from fat), a high-fat diet (HFD, with $60 \%$ of energy from fat), and a high-fat diet blending with 1\% EGCG (HFD + EGCG, with $60 \%$ of energy from fat). Th body weights of the mice of four groups were recorded weekly.

\subsection{Collection of Serum and Tissue Samples}

At the end of the eighth week of the experiment, the serum of the mice was collected after a fast of $12 \mathrm{~h}$ for determining the serum glucose and lipid levels using an automatic biochemical analyzer (TBA-40FR, Toshiba Medical, Tokyo, Japan). Epididymal adipose tissues and hypothalamus samples were collected, immediately frozen in liquid nitrogen, and stored at $-80^{\circ} \mathrm{C}$ for further histology, gene expression, ELISA, and western blot studies.

\subsection{HEE Staining}

Epididymal adipose tissues were washed in saline and fixed with $4 \%$ paraformaldehyde for $24 \mathrm{~h}$ before embedding in paraffin. Embedded adipose tissues were sectioned and stained with hematoxylin and eosin (H\&E), and observed under a microscope (Zeiss, Oberkochen, Germany) at a magnification of $\times 200$.

\subsection{Western Blot Analysis}

BV-2 cells and tissues were lysed in RIPA buffer, containing protease and phosphate inhibitors (Thermo Scientific, Rockford, IL, USA). Protein concentrations in the cell and tissue homogenates were determined using a BCA protein assay kit (Thermo Scientific, Rockford, IL, USA). Equal quantities $(40 \mu \mathrm{g})$ of proteins were separated on a 10\% Sodium Dodecyl Sulfate (SDS)-polyacrylamide gel, 
transferred onto Polyvinylidene Fluoride (PVDF) membranes and blocked with 5\% non-fat milk in TBST for $1 \mathrm{~h}$ at room temperature. The protein abundance was detected after incubating the primary antibodies against STAT3, phospho-STAT3, JAK2, phospho-JAK2, followed by HRP-conjugated secondary antibody. The blots were visualized by an enhanced chemiluminescence (ECL) detection system (Bio-Rad, Hercules, CA, USA).

\subsection{Enzyme-Linked Immunosorbent Assay (ELISA)}

The levels of TNF- $\alpha$, IL- 6 , and IL-1 $\beta$ in culture supernatants and hypothalamic homogenate were measured by ELISA kit (R\&D Systems, Minneapolis, MN, USA) according to the manufacturer's instructional manual, and the OD values were measured at $450 \mathrm{~nm}$. The concentration of each cytokine was calculated from the linear equation derived from the standard curve of the recombinant cytokine in the kit.

\subsection{Immunofluorescence}

Immunofluorescence staining was performed for measuring the expression of Iba- 1 in hypothalamic ARC and PVN as described in our previous research [25]. Ithe mageJ software (NIH, Bethesda, MD, USA) was applied for analyzing the cell number, cell size, and fluorescence intensity.

\subsection{Statistical Analysis}

Data are presented as means \pm standard error of the mean (SEM). Statistical analyses were carried out with one-way ANOVA followed by Tukey's post hoc test using SPSS version 18.0 (IBM Corporation., Armonk, NY, USA). For multiple comparisons, statistical differences among the groups are indicated with superscript letters. Differences between the means were considered statistically significant at $p<0.05$.

Author Contributions: Formal analysis-L.M.; Investigation-L.M., D.H., L.Y., Y.Z. and J.Z.; Methodology-Y.W.; Resources-L.M.; Supervision-Y.W. and P.X.; Writing—original draft-J.Z.; Writing-review \& editing-P.X. All authors have read the manuscript and provided constructive feedback.

Funding: This research received no external funding.

Acknowledgments: The authors are grateful to Yanhong Chen at Zhejiang University Laboratory Animal Center for the technical assistance in checking and conducting animal experiments.

Conflicts of Interest: The authors declare no conflict of interest.

\section{Abbreviations}

$\begin{array}{ll}\text { AgRP } & \text { Agouti-related neuropeptide } \\ \text { ARC } & \text { Arcuate nucleus } \\ \text { CNS } & \text { Central nervous system } \\ \text { DIO } & \text { Diet-induced obesity } \\ \text { EGCG } & (-) \text {-Epigallocatechin gallate } \\ \text { HFD } & \text { High-fat diet } \\ \text { IL-6 } & \text { Interleukin-6 } \\ \text { IL-1 } \beta & \text { Interleukin 1-beta } \\ \text { JAK2 } & \text { Janus kinase 2 } \\ \text { PA } & \text { Palmitic acid } \\ \text { POMC } & \text { Proopiomelanocortin } \\ \text { PVN } & \text { Paraventricular nucleus } \\ \text { SFA } & \text { Saturated fatty acids } \\ \text { STAT3 } & \text { Signal transducers and activators of transcription } 3 \\ \text { TNF- } \alpha & \text { Tumor necrosis factor-alpha }\end{array}$




\section{References}

1. Bray, G.A.; Kim, K.K.; Wilding, J.P.H.; Federation, W.O. Obesity: A chronic relapsing progressive disease process. A position statement of the world obesity federation. Obes. Rev. 2017, 18, 715-723. [CrossRef] [PubMed]

2. Tseng, Y.H.; Cypess, A.M.; Kahn, C.R. Cellular bioenergetics as a target for obesity therapy. Nat. Rev. Drug Discov. 2010, 9, 465-481. [CrossRef] [PubMed]

3. Cooke, A.A.; Connaughton, R.M.; Lyons, C.L.; McMorrow, A.M.; Roche, H.M. Fatty acids and chronic low grade inflammation associated with obesity and the metabolic syndrome. Eur. J. Pharmacol. 2016, 785, 207-214. [CrossRef] [PubMed]

4. De Souza, C.T.; Araujo, E.P.; Bordin, S.; Ashimine, R.; Zollner, R.L.; Boschero, A.C.; Saad, M.J.A.; Velloso, L.A. Consumption of a fat-rich diet activates a proinflammatory response and induces insulin resistance in the hypothalamus. Endocrinology 2005, 146, 4192-4199. [CrossRef] [PubMed]

5. Fang, X.; Ge, K.L.; Song, C.; Ge, Y.L.; Zhang, J.Y. Effects of n-3pufas on autophagy and inflammation of hypothalamus and body weight in mice. Biochem. Biophys. Res. Commun. 2018, 501, 927-932. [CrossRef] [PubMed]

6. Lizarbe, B.; Soares, A.F.; Larsson, S.; Duarte, J.M.N. Neurochemical modifications in the hippocampus, cortex and hypothalamus of mice exposed to long-term high-fat diet. Front. Neurosci. 2019, 12, 985. [CrossRef]

7. Morton, G.J.; Cummings, D.E.; Baskin, D.G.; Barsh, G.S.; Schwartz, M.W. Central nervous system control of food intake and body weight. Nature 2006, 443, 289-295. [CrossRef]

8. Mendes, N.F.; Kim, Y.B.; Velloso, L.A.; Araujo, E.P. Hypothalamic microglial activation in obesity: A mini-review. Front. Neurosci. 2018, 12, 846. [CrossRef]

9. Meydani, M.; Hasan, S.T. Dietary polyphenols and obesity. Nutrients 2010, 2, 737-751. [CrossRef]

10. Zhan, W.; Liu, Y.; Li, D.P.; Liu, Y. Advancing insights on the anti-obesity biochemical mechanism of (-)-epigallocatechin gallate (egcg) by inhibiting alpha-amylase activity. RSC Adv. 2016, 6, 96918-96927. [CrossRef]

11. Martel, J.; Ojcius, D.M.; Chang, C.J.; Lin, C.S.; Lu, C.C.; Ko, Y.F.; Tseng, S.F.; Lai, H.C.; Young, J.D. Anti-obesogenic and antidiabetic effects of plants and mushrooms. Nat. Rev. Endocrinol. 2017, 13, 149-160. [CrossRef] [PubMed]

12. Duffy, C.M.; Yuan, C.; Wisdorf, L.E.; Billington, C.J.; Kotz, C.M.; Nixon, J.P.; Butterick, T.A. Role of orexin a signaling in dietary palmitic acid-activated microglial cells. Neurosci. Lett. 2015, 606, 140-144. [CrossRef] [PubMed]

13. Huang, C.F.; Ma, R.; Sun, S.G.; Wei, G.R.; Fang, Y.; Liu, R.G.; Li, G. Jak2-stat3 signaling pathway mediates thrombin-induced proinflammatory actions of microglia in vitro. J. Neuroimmunol. 2008, 204, 118-125. [CrossRef] [PubMed]

14. Tsunekawa, T.; Banno, R.; Mizoguchi, A.; Sugiyama, M.; Tominaga, T.; Onoue, T.; Hagiwara, D.; Ito, Y.; Iwama, S.; Goto, M.; et al. Deficiency of ptp1b attenuates hypothalamic inflammation via activation of the jak2-stat3 pathway in microglia. Ebiomedicine 2017, 16, 172-183. [CrossRef] [PubMed]

15. de Git, K.C.G.; Adan, R.A.H. Leptin resistance in diet-induced obesity: The role of hypothalamic inflammation. Obes. Rev. 2015, 16, 207-224. [CrossRef] [PubMed]

16. Thaler, J.P.; Guyenet, S.J.; Dorfman, M.D.; Wisse, B.E.; Schwartz, M.W. Hypothalamic inflammation: Marker or mechanism of obesity pathogenesis? Diabetes 2013, 62, 2629-2634. [CrossRef] [PubMed]

17. Thaler, J.P.; Schwartz, M.W. Minireview: Inflammation and obesity pathogenesis: The hypothalamus heats up. Endocrinology 2010, 151, 4109-4115. [CrossRef]

18. Valdearcos, M.; Douglass, J.D.; Robblee, M.M.; Dorfman, M.D.; Stifler, D.R.; Bennett, M.L.; Gerritse, I.; Fasnacht, R.; Barres, B.A.; Thaler, J.P.; et al. Microglial inflammatory signaling orchestrates the hypothalamic immune response to dietary excess and mediates obesity susceptibility. Cell Metab. 2018, 27, 1356. [CrossRef]

19. Santos, C.; de Lima, C.M.; Foro, C.A.R.; de Oliveira, M.A.; Magalhaes, N.G.M.; Guerreiro-Diniz, C.; Diniz, D.G.; Vasconcelos, P.F.D.; Diniz, C.W.P. Visuospatial learning and memory in the cebus apella and microglial morphology in the molecular layer of the dentate gyrus and ca1 lacunosum molecular layer. J. Chem. Neuroanat. 2014, 61-62, 176-188. [CrossRef]

20. Bollinger, J.L.; Collins, K.E.; Patel, R.; Wellman, C.L. Behavioral stress alters corticolimbic microglia in a sexand brain region-specific manner. PLOS ONE 2017, 12, e0187631. [CrossRef] 
21. Konstantinidi, M.; Koutelidakis, A.E. Functional foods and bioactive compounds: A review of its possible role on weight management and obesity's metabolic consequences. Medicines 2019, 6, 94. [CrossRef] [PubMed]

22. Friedrich, M.; Petzke, K.J.; Raederstorff, D.; Wolfram, S.; Klaus, S. Acute effects of epigallocatechin gallate from green tea on oxidation and tissue incorporation of dietary lipids in mice fed a high-fat diet. Int. J. Obes. 2012, 36, 735-743. [CrossRef] [PubMed]

23. Grove, K.A.; Sae-tan, S.; Kennett, M.J.; Lambert, J.D. (-)-epigallocatechin-3-gallate inhibits pancreatic lipase and reduces body weight gain in high fat-fed obese mice. Obesity 2012, 20, 2311-2313. [CrossRef] [PubMed]

24. Jang, H.J.; Ridgeway, S.D.; Kim, J.A. Effects of the green tea polyphenol epigallocatechin-3-gallate on high-fat diet-induced insulin resistance and endothelial dysfunction. Am. J. Physiol. Metab. 2013, 305, E1444-E1451. [CrossRef] [PubMed]

25. Zhou, J.H.; Mao, L.M.; Xu, P.; Wang, Y.F. Effects of (-)-epigallocatechin gallate (egcg) on energy expenditure and microglia-mediated hypothalamic inflammation in mice fed a high-fat diet. Nutrients 2018, 10. [CrossRef] [PubMed]

26. Atrens, D.M.; Menendez, J.A. Glucagon and the paraventricular hypothalamus-modulation of energy-balance. Brain Res. 1993, 630, 245-251. [CrossRef]

27. Carvalheira, J.B.C.; Ribeiro, E.B.; Araujo, E.P.; Guimaraes, R.B.; Telles, M.M.; Torsoni, M.; Gontijo, J.A.R.; Velloso, L.A.; Saad, M.J.A. Selective impairment of insulin signalling in the hypothalamus of obese zucker rats. Diabetologia 2003, 46, 1629-1640. [CrossRef]

28. Yi, C.X.; Tschop, M.H.; Woods, S.C.; Hofmann, S.M. High-fat-diet exposure induces igg accumulation in hypothalamic microglia. Dis. Model. Mech. 2012, 5, 686-690. [CrossRef]

29. Cunningham, C. Microglia and neurodegeneration: The role of systemic inflammation. Glia 2013, 61, 71-90. [CrossRef]

30. He, L.X.; Tong, X.Y.; Zeng, J.; Tu, Y.Q.; Wu, S.C.; Li, M.P.; Deng, H.M.; Zhu, M.M.; Li, X.C.; Nie, H.; et al. Paeonol suppresses neuroinflammatory responses in lps-activated microglia cells. Inflammation 2016, 39, 1904-1917. [CrossRef]

31. Stentz, F.B.; Kitabchi, A.E. Palmitic acid-induced activation of human t-lymphocytes and aortic endothelial cells with production of insulin receptors, reactive oxygen species, cytokines, and lipid peroxidation. Biochem. Biophys. Res. Commun. 2006, 346, 721-726. [CrossRef] [PubMed]

32. Hidalgo-Lanussa, O.; Avila-Rodriguez, M.; Baez-Jurado, E.; Zamudio, J.; Echeverria, V.; Garcia-Segura, L.M.; Barreto, G.E. Tibolone reduces oxidative damage and inflammation in microglia stimulated with palmitic acid through mechanisms involving estrogen receptor beta. Mol. Neurobiol. 2018, 55, 5462-5477. [CrossRef] [PubMed]

33. Harrison, L.; Pfuhlmann, K.; Schriever, S.C.; Pfluger, P.T. Profound weight loss induces reactive astrogliosis in the arcuate nucleus of obese mice. Mol. Metab. 2019, 24, 149-155. [CrossRef] [PubMed]

34. Lee, C.H.; Kim, H.J.; Lee, Y.S.; Kang, G.M.; Lim, H.S.; Lee, S.H.; Song, D.K.; Kwon, O.; Hwang, I.; Son, M.; et al. Hypothalamic macrophage inducible nitric oxide synthase mediates obesity-associated hypothalamic inflammation. Cell Rep. 2018, 25, 934-946. [CrossRef]

35. Arruda, A.P.; Milanski, M.; Coope, A.; Torsoni, A.S.; Ropelle, E.; Carvalho, D.P.; Carvalheira, J.B.; Velloso, L.A. Low-grade hypothalamic inflammation leads to defective thermogenesis, insulin resistance, and impaired insulin secretion. Endocrinology 2011, 152, 1314-1326. [CrossRef] [PubMed]

36. Liu, Z.T.; Chen, T.C.; Lu, X.X.; Xie, H.Y.; Zhou, L.; Zheng, S.S. Overexpression of variant pnpla3 gene at i148m position causes malignant transformation of hepatocytes via il-6-jak2/stat3 pathway in low dose free fatty acid exposure: A laboratory investigation in vitro and in vivo. Am. J. Trans. Res. 2016, 8, 1319-U1341.

37. Ahmad, S.F.; Attia, S.M.; Bakheet, S.A.; Zoheir, K.M.A.; Ansari, M.A.; Korashy, H.M.; Abdel-Hamied, H.E.; Ashour, A.E.; Abd-Allah, A.R.A. Naringin attenuates the development of carrageenan-induced acute lung inflammation through inhibition of nf-kappa $b$, stat 3 and pro-inflammatory mediators and enhancement of $i$ kappa b alpha and anti-inflammatory cytokines. Inflammation 2015, 38, 846-857. [CrossRef]

38. Al-Massri, K.F.; Ahmed, L.A.; El-Abhar, H.S. Pregabalin and lacosamide ameliorate paclitaxel-induced peripheral neuropathy via inhibition of jak/stat signaling pathway and notch-1 receptor. Neurochem. Int. 2018, 120, 164-171. [CrossRef]

39. Bates, S.H.; Stearns, W.H.; Dundon, T.A.; Schubert, M.; Tso, A.W.K.; Wang, Y.P.; Banks, A.S.; Lavery, H.J.; Haq, A.K.; Maratos-Flier, E.; et al. Stat3 signalling is required for leptin regulation of energy balance but not reproduction. Nature 2003, 421, 856-859. [CrossRef] 
40. Wang, Y.; Zang, W.; Ji, S.; Cao, J.; Sun, C. Three polymethoxyflavones purified from ougan (citrus reticulata cv. Suavissima) inhibited lps-induced no elevation in the neuroglia bv-2 cell line via the jak2/stat3 pathway. Nutrients 2019, 11, 791. [CrossRef]

41. Wang, Y.; Ji, S.; Zang, W.; Wang, N.; Cao, J.; Li, X.; Sun, C. Identification of phenolic compounds from a unique citrus species, finger lime (citrus australasica) and their inhibition of lps-induced no-releasing in bv-2 cell line. Food Chem. Toxicol. 2019, 129, 54-63. [CrossRef] [PubMed]

42. Ibars, M.; Ardid-Ruiz, A.; Suarez, M.; Muguerza, B.; Blade, C.; Aragones, G. Proanthocyanidins potentiate hypothalamic leptin/stat3 signalling and pomc gene expression in rats with diet-induced obesity. Int. J. Obes. 2017, 41, 129-136. [CrossRef] [PubMed]

43. Kou, X.J.; Qi, S.M.; Dai, W.X.; Luo, L.; Yin, Z.M. Arctigenin inhibits lipopolysaccharide-induced inos expression in raw264.7 cells through suppressing jak-stat signal pathway. Int. Immunopharmacol. 2011, 11, 1095-1102. [CrossRef] [PubMed]

44. Wang, Z.; Liu, D.X.; Wang, F.W.; Liu, S.M.; Zhao, S.D.; Ling, E.A.; Hao, A.J. Saturated fatty acids activate microglia via toll-like receptor 4/nf-kappa b signalling. Br. J. Nutr. 2012, 107, 229-241. [CrossRef] [PubMed]

(C) 2019 by the authors. Licensee MDPI, Basel, Switzerland. This article is an open access article distributed under the terms and conditions of the Creative Commons Attribution (CC BY) license (http://creativecommons.org/licenses/by/4.0/). 